\title{
A Wearable Inertial Sensor System for Human Motion Analysis
}

\author{
Tao Liu, Yoshio Inoue, Kyoko Shibata \\ Department of Intelligent Mechanical Systems Engineering \\ Kochi University of Technology \\ 185 Miyanokuchi, Tosayamada-cho, Kochi Japan 782-8502 \\ 078008g@gs.kochi-tech.ac.jp
}

\begin{abstract}
To analyze the multi-dimension motion of human foot during walking, a wearable inertial sensor system is proposed. In this system, two gyroscopes (ENC-05EB) were used to measure angular velocities in $x$ - and $y$ - directions, and an accelerometer (ADXL202) was used to measure acceleration in $\mathrm{x}$ - and $\mathrm{y}$ - directions respectively. Furthermore, a fuzzy inference system (FIS) was developed for the calculation of the gait phases derived from sensors' outputs. A digital filter was also designed to remove noises from of the output of the fuzzy inference system, which enhances robustness of the system. Finally, experimental study was conducted, and a wearable mechanism with the sensors system was constructed to detect gait phases of the normal human walking.
\end{abstract}

Index Terms-human motion analysis; motion sensor; gait phases; fuzzy inference.

\section{INTRODUCTION}

Gait analyses have been found in many application fields: functional electrical stimulation (FES) for restoring patents' walking function; human dynamic analysis system to finish force and motion analysis, when some force sensors are integrated; humanoid robot including assistant mechanism for walking. In daily life, human dynamics analysis is becoming important, for example, the gait analysis of pregnant woman can illustrate the different body dynamic conditions that ankle joint moment and hip joint moment are much larger than non-pregnant women. Based on gait phase analysis, the assistant and rehabilitative devices can be developed for pregnant woman or patients [1].

The integration of three-dimension motion measurement using multi-camera system and ground reaction force measurement using force plates has been successfully devoted to tracking human body parts and performing dynamics analysis of their physical behaviors in a complex environment [2][3]. However this method needs sizeable work space and high-speed graphic signal processing devices, therefore it is inefficient in real time controls and is too expensive for daily product developments. Moreover, human body is composed of many highly flexible segments, and the up-body motion of human is especially complicated for accuracy calculations. Thus, in the development of cheaper and more comfortable gait analysis device, several sensor combinations, including force sensitive resistors, inclinometer, goniometers, gyroscopes and accelerometers, were proposed to perform

\author{
Xueyan Tang \\ School of Mechanical and Production Engineering \\ Nanyang Technological University \\ Singapore 639798 \\ TANG0031@ntu.edu.sg
}

gait phases analysis. In the study by $\mathrm{S} . \mathrm{K} . \mathrm{Ng}$ and $\mathrm{H}$. J. Chizeck [4], measurements from goniometers at the hip, knee and ankle joints were used in combination with a fuzzy model classification method to detect five different gait phases. However, the method suffered from frequency detection errors, and the measure devices also make human feel uncomfortable. In [5], K. Tong and H. M. Grant proposed a measurement device using two gyroscopes, one placed on the thigh and the other one on the shank, which can estimate knee rotation angle during walking. This system can detect difference phases of human walking, but the dispersed measurement system may affect user walking In [6], R. Willemsen et al. proposed a detection system using accelerometers, but this system just can separate stance phase and swing phase from the human walking gait phases. Ion P.I. Pappas al. in [7] used a detection system consisting of three force sensitive resistors that measure the force loads on a shoe insole and a miniature gyroscope chip that measures the rotational velocity of the foot. The system detects accurately and reliably the four gait phases in real time, but it was just designed for the application of functional electrical stimulation

To finish the 3-D human dynamic analysis by using some wearable devices, in our study, we have developed a system composed of a wearable motion analysis system and a wearable 6-D force sensor. We will use the developed 6-D force sensor [8] to measure reaction forces and moments in three directions, and use the motion analysis system to detect 3-D motion of human motion during walking. The results of motion analysis and force analysis will be combined to finish dynamic analysis of human's walking. In this paper, we focus on the design of motion analysis system for gait analysis during human's walking.

The motion analysis system was designed as a different sensors combination consisting of two gyroscope-chips that measure two-direction rotational velocities of the foot and a two-axis accelerometer-chip that can output twodirection accelerations of the foot during walking. Furthermore, a fuzzy inference system (FIS) was developed for the analysis of sensors' outputs, which can estimate a gait phase analysis result. To get the decided gait phases change points of human walking, a digital filter was used to remove noises from the outputs of the fuzzy inference system. Finally, experimental study was conducted. A wearable mechanism with the sensors system 
was constructed, and some experiments about detection gait phases of the normal human walking were finished.

\section{MeTHODS AND MATERIALS}

\section{A. Hardware Description of the Inertial Sensor System}

The gait analysis system performs the detection of gait phases by using two types of inertial sensors, i.e., two gyroscopes used to measure angular velocities of two-axis on the foot plane during walking, and a two-axis accelerometer used to measure total transmission accelerations including gravity acceleration and dynamic acceleration along two sensitive axis.

As shown in Fig. 1, an electrical base board was designed for the inertial sensor system. Two miniature gyroscopes (Murata ENC-03J, size $15.5 \times 8.0 \times 4.3 \mathrm{~mm}$, weight $10 \mathrm{~g}$ ) were integrated on the base board with their sensing axis oriented on the bottom plane of the foot respectively. The two gyroscopes can measure twodimension rotations of the foot in that plane. The Murata ENC-03J gyroscope measures the rotational velocity by sensing the mechanical deformation caused by the Coriolis force on an internal vibrating prism. The gyroscope signal is filtered by a third-order band-pass filter $(0.25-25 \mathrm{~Hz})$ with a $20-\mathrm{dB}$ gain in the pass band. The frequencies outside the pass-band were filtered out because they are not related to the walking kinetics. The filtered gyroscope signal was used to directly estimate the angular velocity of the foot and it was integrated to estimate the inclination of the foot relative to the ground. The accelerometer-chip with almost the same theory as gyroscope-chip was fixed on the back of the base board, which can measure two-axis accelerations including gravity acceleration and dynamic acceleration during walking. In the design of data record device, the sampling time of $\mathrm{A} / \mathrm{D}$ module is selected according to the bandwidth of signals, and the sampling frequency should be higher than $2 \times 1.25 \times 25 \mathrm{~Hz}$ (the bandwidth of the inertial sensors' signals is about $25 \mathrm{~Hz}$ ) [9]. Therefore the sensor signals were sampled at a frequency of $100 \mathrm{~Hz}>62.5 \mathrm{~Hz}$ with a resolution of 14 bits through A/D card (Keyence NR-110), and the sample data is saved into the person computer for the analysis. The card is connected with computer through micro-card interface of PCMCIA2.1 in personal computer. Because the two kinds of inertial sensors are low energy consumed electrical devices, the motion analysis system is powered by using two button batteries (CR2032), which can work up to 30 minutes in walking experiments.

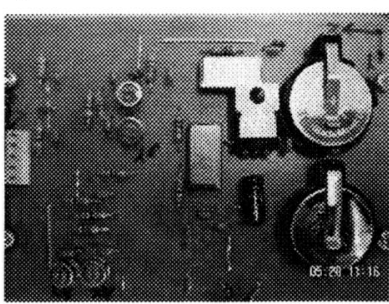

(a) Front of the board

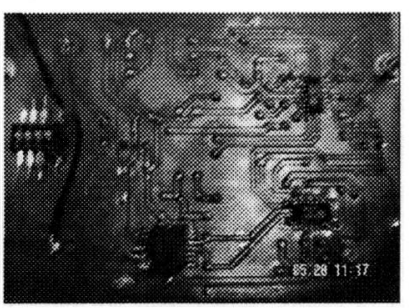

(b) Back of the board
Fig. 1 Base board of the sensor system

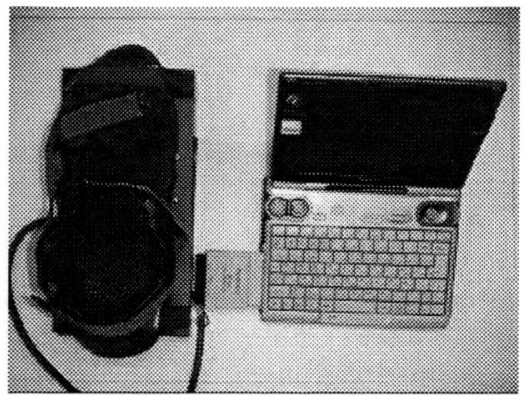

Fig. 2 Hardware device of the motion anlysis system

A mechanical shoe (see Fig. 2) was designed to fix the base board of inertial sensors. The foot plane is parallel to the base board. The material was selected as Aluminum, and the weight is about $500 \mathrm{~g}$, the size is almost the same as common shoes.

\section{B. Physics Sense Analysis of Gait Phases}

In this paper, a normal walking gait cycle is divided into four different gait phases, i.e., stance, toe-rotation, swing, and heel-rotation. The following is the definition of the gait phases (see Fig. 3): let stance phase be the period when the foot is with its entire length in contact with the ground; let toe-rotation phase be the period following the stance phase during which the front part of the foot is in contact with the ground and its heel is rotating around toe joint; let swing phase be the period when the foot is in the air (not in contact with the ground); let heel-rotation phase be the period following the swing phase which begins with the first contact of the foot with the ground (usually the heel, but not necessarily) and the front part of foot is rotating around heel's contacting point, which ends when the entire foot touches the ground.

We define a walking cycle as the period from one stance phase of the foot to the next stance phase of the same foot. In gait phase analysis algorism, these gait phases were represented by a mathematics method with four distinct value ranges. The loop frequency of the phase record was $100 \mathrm{~Hz}$, i.e., equal to the sensors sampling frequency.

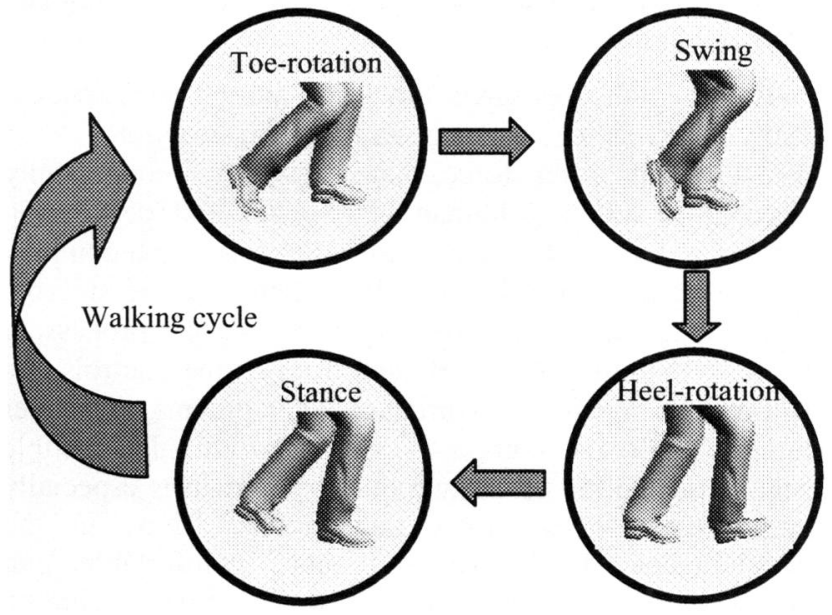

Fig. 3 Transition of four gait phases 


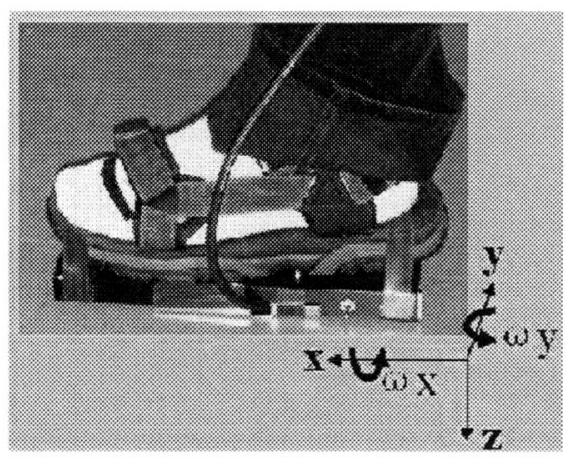

Fig. 4 Wearable motion analsys mechanical device

Physics sense analysis of each phase was performed to prepare design rules for the gait phase detection algorithm. As shown in Fig. 4, when the motion measure device wears under the foot, we suppose the subject is viewed from the lateral side and clockwise rotations are considered positive. The $\mathrm{Z}$ - axis is vertical to the foot plane, and $\mathrm{X}$ - axis and $\mathrm{Y}$ - axis are along length- and wideorientations of foot respectively. The symbols $\omega_{x}$ and $\omega_{y}$ represent the rotational velocity of foot around $\mathrm{X}$ - axis and Y- axis respectively. Physics senses of each phase can be defined as following:

1). If $\omega_{\mathrm{x}}=0$ AND $\omega_{\mathrm{y}}=0$ AND $\mathrm{A}_{\mathrm{x}}=0$ AND $\mathrm{A}_{\mathrm{y}}=0$ Then 'Stance Phase';

2). If $\omega_{y}<0$ AND $A_{x} \neq 0$ AND $A_{y} \neq 0$ Then 'Swing Phase';

3). If $\omega_{y}>0$ AND $A_{x} \neq 0$ AND $A_{y} \neq 0$ Then If the case is before the 'Swing Phase' of the same walking cycle Then 'Toe-rotation Phase' Else 'Heel-rotation Phase'

\section{Design of a Fuzzy Inference System}

When the experiments data are recorded in the hard-disk of personal computer, the off-line analysis is made to analyze the gait during walking. The inertial sensors output signals are easy to be affected by interrupts from testing environmental noise, and the static float of the inertial sensors can decrease the precision of the measurements in the case of long time testing, so in the study, a fuzzy inference system (FIS) is proposed to improve precision of the detection of gait phase. The fuzzy system is robust to the noise from the inertial sensors. We design the fuzzy inference system by using software of MATLAB (see Fig. 5). The two gyroscopes' outputs and the accelerometer's two-axis outputs are defined as the four inputs of the FIS, and the output of FIS is a value of gait phases.

In the design of fuzzy inference system, Mamdani fuzzy inference method was used as the inference method. Each fuzzy input was defined as three fuzzy ranges: negative, zero and positive (see Fig. 6), and each fuzzy range was designed by using saw-tooth function. In the same way, the output of FIS was defined as four fuzzy ranges to de-fuzzy inference results in the Mamdani method. As shown in Fig. 7, the four output ranges is named as: stand, toe-r, swing, and heel-r respectively.

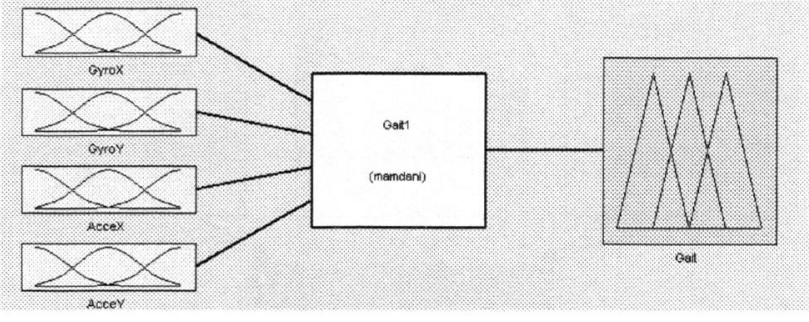

Fig. 5 Theory schematics of theFIS

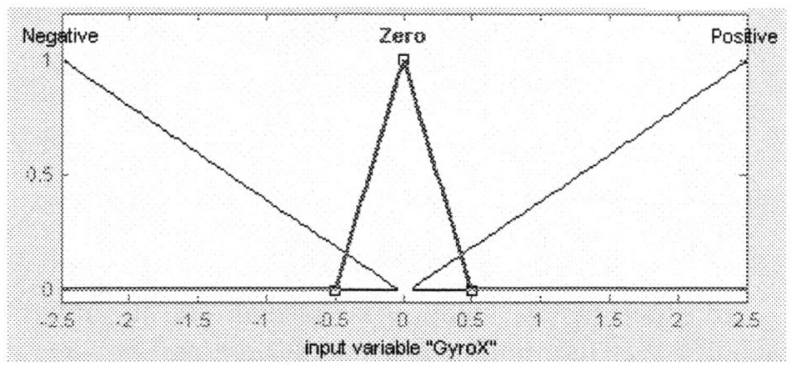

Fig. 6 Fuction of fuzzy input

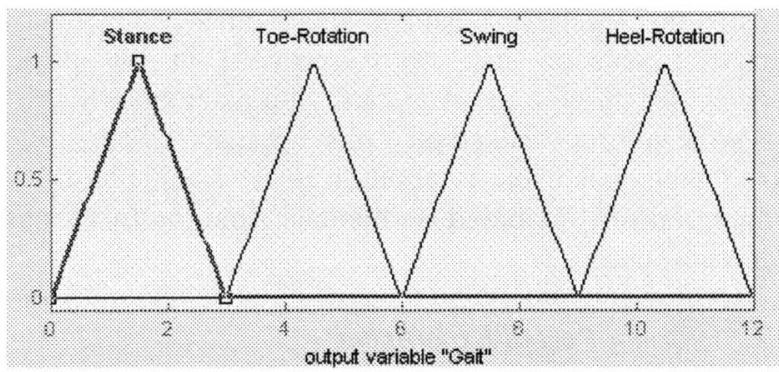

Fig. 7 De-fuzzy output of the FIS

\section{Design of a Digital Filter}

In this section, a digital filer designed for the outputs of FIS is presented. The inertial sensors are sensitive to the environmental noise, which leads to the difficulty of detecting the gait phases precisely using a simple algorithm in a micro-computer. To get the decided gait phases change points of human walking, a digital filter is used to remove noise results from the fuzzy inference system. We recorded the inertial sensor system's signals at a frequency of $100 \mathrm{~Hz}$, and the normal human walking period is about $1.5 \mathrm{sec}$, so in the results of FIS the pulse interrupts with period of no over $0.05 \mathrm{sec}$ can be confirmed as the errors pulses in the gait phase detection. A digital filter was designed to filter the error pulses in the results of FIS. The symbols $\mathrm{R}(\mathrm{i})$ and $\mathrm{Rf}(\mathrm{i})$ represent the output result of FIS and last filtered results on the $i$-th sampling cycle $(\mathrm{i}=2,3,4 \ldots)$, and $\mathrm{k}$ represents the noise pulse swing value. Then the rule of the filter is designed as follows. If the absolute value of $R(i-1)$ subtracted by $R(i+3)$ is far no more than $\mathrm{k}$, and one of the absolute value among $\mathrm{R}(\mathrm{i})$ subtracted by $\mathrm{R}(\mathrm{i}-1)$, $\mathrm{R}(\mathrm{i}+1)$ subtracted by $\mathrm{R}(\mathrm{i}-1)$ and $R(i+2)$ subtracted by $R(i-1)$ is larger than $k$, then the value of $R(i), R(i+1)$ and $R(i+2)$ is set as the same value as $R(i-$ 1 ), because in this case the $50 \mathrm{~ms}$ sampling range (from $\mathrm{i}-1$ to $\mathrm{i}+3$ ) must be added in a noise pulse (see Fig. 8). 


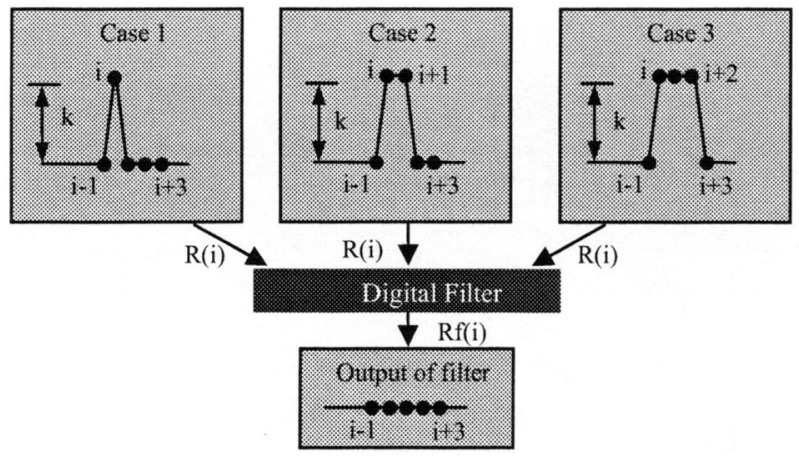

Fig. 8 Theory of the digital filter

\section{EXPERIMENTAL STUDY}

An experimental study was carried out in order to quantify that the motion analysis system can be used on normal gait phases detection for the future motion analysis study. The study involved a group of healthy adults who worn the special mechanical shoes on the one foot. The inertial sensors' data were recorded in the personal computer through the A/D card with resolution of 14 bits. All the subjects walked on a plane ground (length $=30 \mathrm{~m}$ ), and their gait cycle is about $1.5 \mathrm{~s}$. The gait phases were analyzed off-line based on the data from the two kinds of inertial sensors.

\section{A. Formal Part of Experiment}

After calibrated to be zero when no motion and adjusted to change from -2.5 to 2.5 in the same range, the four-output data from inertial sensors were fed into the fuzzy inference system according to sampling time cycle. As shown in Fig. 9, one subject's motion data were recorded about using $\mathrm{x}$ - axis gyroscope, $\mathrm{y}$ - axis gyroscope, $\mathrm{x}$ - axis accelerometer, $\mathrm{y}$ - axis accelerometer respectively.

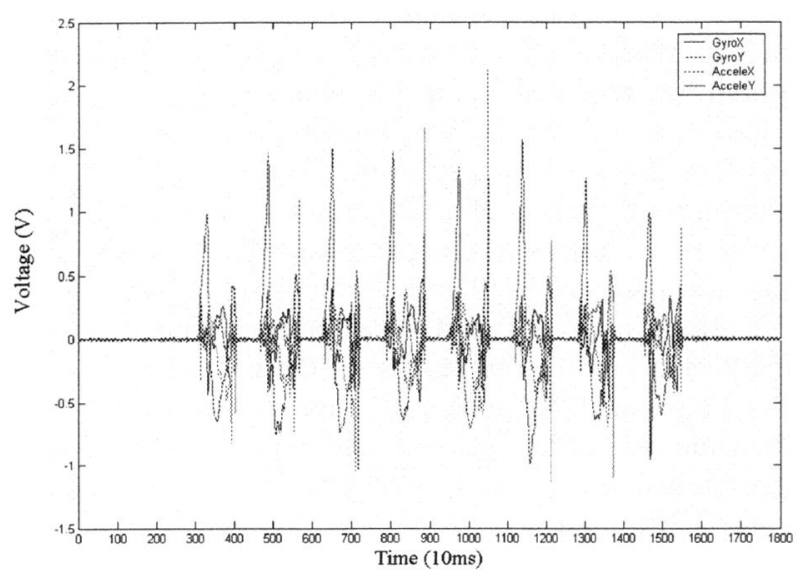

Fig. 9 Four recorded signals of the inertial sensors

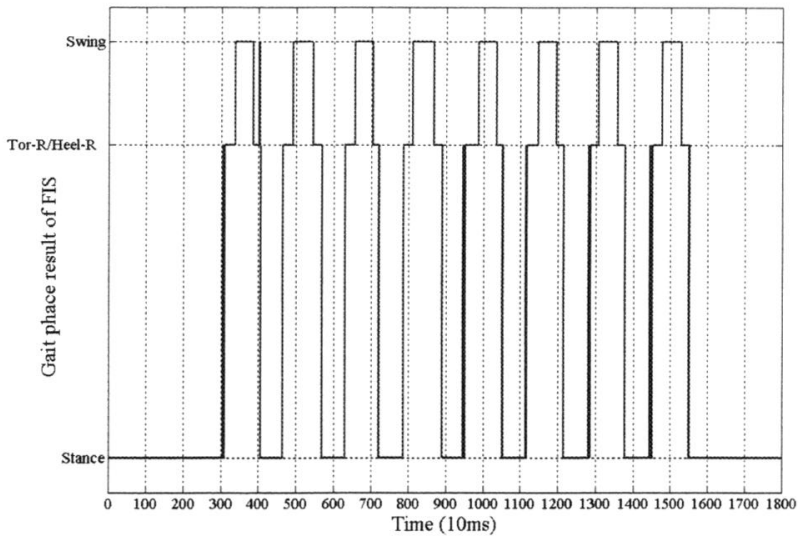

Fig. 10 Inference result of the FIS

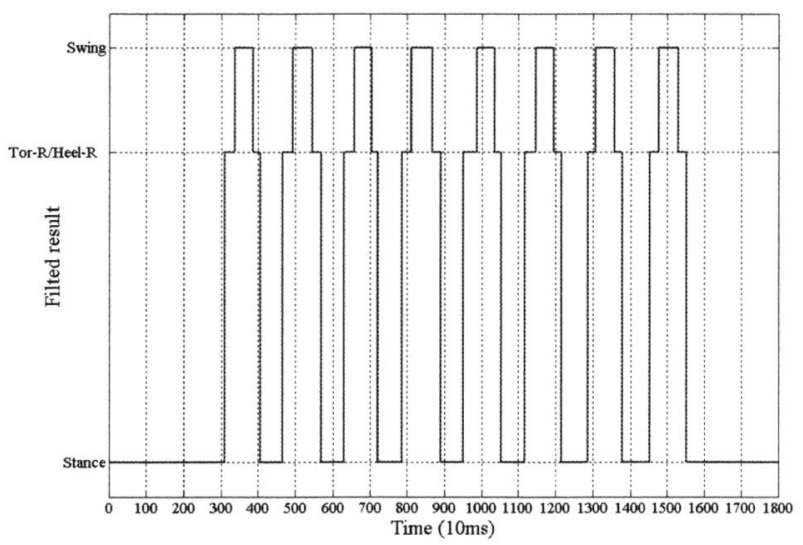

Fig. 11 Filtered inference result of the FIS

Figure 10 shows the inference result graph of the fuzzy inference system, in which the stance phase and swing phase can be clearly divided and the toe-rotation phase and the heel-rotation phase are separated by the swing phase. However, the noise pulses in the result graph make it is difficult that the real separate points of each gait phase are detected from the reference results.

\section{B. Post-part of Experiment}

In this section, the post-process of inference results is presented. A digital filter was designed to filter the error pulses in the results of FIS. Figure 11 shows the filtered results of gait phase analysis algorithm. In the analysis, the sampling cycle is $10 \mathrm{~ms}$, and table 1 lists the sampling numbers of every transition point in the all walking cycle. The object's gait phase analysis results graph was regenerated to prepare for the motion analysis of walking in the future study. 


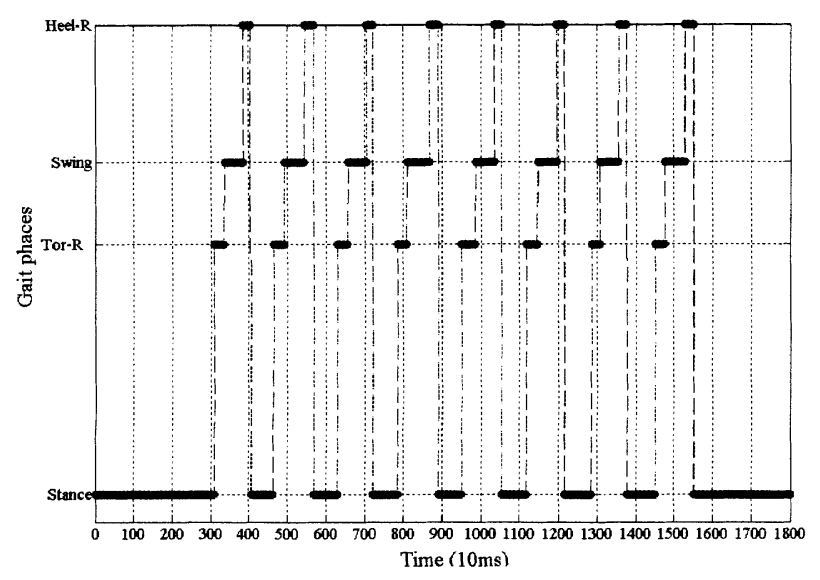

Fig. 12 Gait phase analysis result of a object

TABLE I.GAIT PHASES TRANSITION POINTS OF AN OBJECT

\begin{tabular}{|c|c|c|c|c|}
\hline \multirow{2}{*}{$\begin{array}{c}\text { Walking } \\
\text { cycle } \\
\text { number }\end{array}$} & \multicolumn{4}{|c|}{ Transition time (10ms) } \\
\cline { 2 - 5 } & $\begin{array}{c}\text { Stance to } \\
\text { Toe-r }\end{array}$ & $\begin{array}{c}\text { Toe-r to } \\
\text { Swing }\end{array}$ & $\begin{array}{c}\text { Swing to } \\
\text { Heel-r }\end{array}$ & $\begin{array}{c}\text { Heel-r to } \\
\text { Stance }\end{array}$ \\
\hline 1 & 309 & 336 & 385 & 404 \\
\hline 2 & 464 & 493 & 544 & 568 \\
\hline 3 & 629 & 656 & 705 & 721 \\
\hline 4 & 785 & 809 & 867 & 890 \\
\hline 5 & 950 & 985 & 1034 & 1053 \\
\hline 6 & 1117 & 1145 & 1195 & 1215 \\
\hline 7 & 1285 & 1307 & 1356 & 1376 \\
\hline 8 & 1451 & 1476 & 1529 & 1551 \\
\hline
\end{tabular}

\section{CONCLUSION}

A different motion analysis sensor that reliably identified the gait phase transitions between stance, toerotation, swing, and heel-rotation, was presented. This offline analysis system was based on a set of inertial sensors combination including two miniature gyroscopes and a miniature two-axis accelerometer, which were integrated on a based board to be worn under foot. The sensor signals were sampled at a frequency of $100 \mathrm{~Hz}$ with a resolution of 14 bits through $\mathrm{A} / \mathrm{D}$ card, and the sample data is fed into the person computer for the off-line analysis. A fuzzy inference system (FIS) was designed to improve precision of the detection of gait phase. A digital filter is used to remove noise results from the outputs of fuzzy inference system. In experimental study, the gait phase detections were finished successfully on a group of healthy objects.

In the future, the results of gait phase analysis can be used for multi-dimension motion analysis of walking, and a dynamic analysis of walking can be finished when a reactions force measurement system is integrated into this analysis system.

\section{REFERENCES}

[1] Gerald F. Harris, Peter A. Smith, "Human Motion Analysis," Reading, IEEE, Inc., 1996.

[2] I.A. Karaulova, P.M. Hall, A.D. Marshall, "Tracking people in three dimensions using a hierarchical model," Elsevier Science, Image and Vision Computing, Vol.20, pp.691-700, 2002.

[3] Yoshio Inoue, Takuya Matsuda, Kyoko Shibata, "Estimation of vertical reaction force and ankle joint moment by using plantar pressure sensor," JSME, Symposium on human dynamics, pp. 57-62, 2003. (In Japanese)

[4] S. K. Ng and H. J. Chizeck, "Fuzzy model identification for classification of gait events in paraplegics," IEEE Trans. Fuzzy Syst., vol. 5, pp 536-544, Aug. 1997.

[5] K. Tong and H. M. Granat, "A practical gait analysis system using gyroscopes," Med. Eng. Phys., vol. 21, pp. 87-94, 1999.

[6] R. Williamson and B. J. Andrews, "Gait event detection for FES using accelerometers and supervised machine learning," IEEE Trans. Rehab. Eng., vol. 8, pp. 312-319, June 2000.

[7] Ion P. I. Pappas, Milos R. Popovic, Thierry Keller, Volker Dietz, and Manfred Morari, "A reliable gait phase detection system," IEEE Trans. Rehab. Eng., vol. 9, No. 2, pp. 113-125, June 2001.

[8] Tao Liu, Yoshio Inoue, Kyoko Shibata, Yohei Yamasaki, Masafumi Nakahama, "A Six-dimension Parallel Force Sensor for Human Dynamics Analysis," Proceedings of the 2004 IEEE Conference on Robotics, Automation and Mechatronics Singapore, pp. 208-212, 2004

[9] S. M. Sze "Semiconductor Sensors. Wiley," New-York, USA, 1994 